\title{
Evaluating the Performance of MPLS and Frame-Relay using OPNET Modeler
}

\author{
Shahad H. Zwayen \\ Iraq, Baghdad \\ Al-Nahrain University
}

\author{
Mustapha B. Ibrahim \\ Iraq, Baghdad \\ Al-Nahrain Univesity
}

\begin{abstract}
Multiprotocol label Switching (MPLS) technique is one of the latest technologies, which is escalating dramatically because of the great benefits especially in the field of VPN. It is now progressing very quickly to be the number one technology for connecting networks of service providers around the world. In this research, we'll be conducting a comparison between MPLS and Frame-Relay and compare the results between them using OPNET Modeler 14.5 by using video conferencing as a traffic load generator for the network .
\end{abstract}

\section{General Terms}

End-to-End, delay, traffic received.

\section{Keywords}

MPLS, Frame-Relay, OPNET

\section{INTRODUCTION}

Due to the progress in the field of information technology and the importance of it in how to transfer data from the sender to the recipient in the shortest possible time while retaining the high quality of performance technology .We are going to discuss namely MPLS for working on it and that has begun to replace the old technologies such as Frame-Relay. The importance of MPLS is to offer many advantages to the service providers while maintaining high quality, less cost and working to reduce routing tables, as well as making the network providers almost free service protocol Border Gateway Protocol (BGP), which is one of the most important protocols causing high pressure on the device, like routers or any other device [1-2]. MPLS technology provides great services with respect to Layer 3 Virtual Private Network (VPN). MPLS forward data based on Labels instead of layer 3 forwarding which makes network forwarding more efficient and takes care about Interior Gateway Protocol (IGP) like OSPF which is weirdly recommenced for service providers because it's an open standard provided by IEEE [3-4].In other hand Frame-Relay is a method of packet switching. The switching packets networks enable end stations to dynamically share the network medium and the available bandwidth. The following two Variable-length packets and Statistical multiplexing Variable-length packets are used for more efficient and flexible data transfers. These packets are switched between the various segments in the network until the destination is reached. Statistical multiplexing techniques control network access in a packet-switched network. The advantage of this technique is to accommodate more flexibility and more efficiency use of bandwidth. Most of today's popular LANs, such as Ethernet and Token Ring, are packet-switched networks. The two main disadvantages of Frame-Relay are slowing down due to network congestion and difficulties in ensuring Quality of Service (QoS). All service providers Frame Relay customers use a collective network, there can be times when data transmission outstrips network capacity. The difficulty in ensuring QoS is due to the fact that Frame Relay uses variable-length packets. It is easier to guarantee QoS when using a fixed-length packet.ATM (Asynchronous Transfer Mode) involve a fixed-length packet (called a cell in ATM terminology) where Frame Relay uses variable-length packets. Using fixed-length cells that make QoS calculations much more straightforward. Good QoS is important in applications like voice and video conferencing that cannot tolerate significant network delays. [5]. To be up to today's researches we will compare between the two technologies mentioned above Using a single simulation program, we used OPNET Modeler 14.5 where we will set up a group of routers, which represents a network service provider that uses this technique to provide services that will be built on the basis of comparison to use video-conferences over the network.

\section{RELATED WORK}

Because MPLS is one of the most recent technologies in the world, service providers have become more interested to study and prepare to change the infrastructure to become compatible with it. As a comparison between MPLS and Frame-relay using OPNET tool is roared to find. We tried to study the features that were mentioned in other articles that are related to both technologies. In article[6] which talked about MPLS using traffic engineering and briefly discuss it with other technologies such as ATM and Frame-Relay. While article[7] talked about Frame-Relay as a layer 2 VPN compared with layer 3 VPN using MPLS.

\section{EXPERIMENTAL SETUP}

In order to study the performance of core service provider networks, the network setup have been simulated using OPNET 14.5 simulator and various tests on it have been conducted. Performance of the proposed system is evaluated with different scenarios. Our project is implementing a core service provider based on MPLS technology that we found from our research and simulations that MPLS is better and faster than frame-relay and other legacy technologies such as ATM. We suggested a network service provider that we had implemented in both MPLS and Frame-Relay technologies as shown in figure 1 and figure 2. 




Fig.1: MPLS Topology

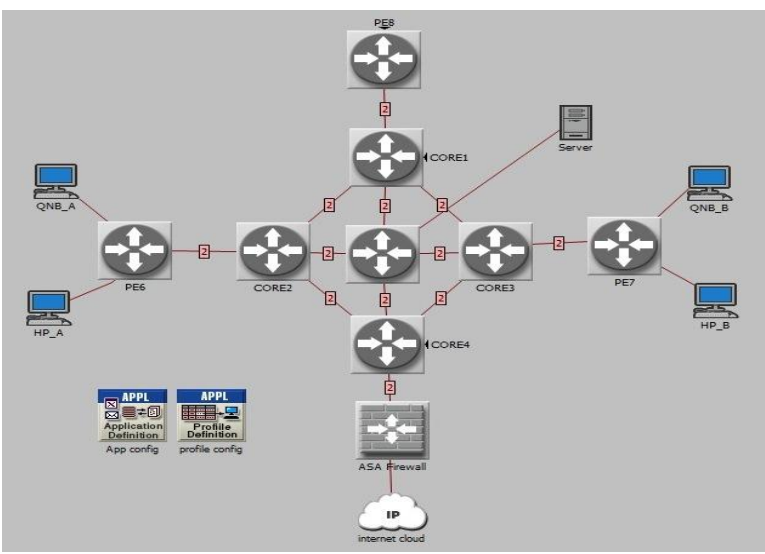

Fig.2: Frame-Relay Topology

\subsection{Delay}

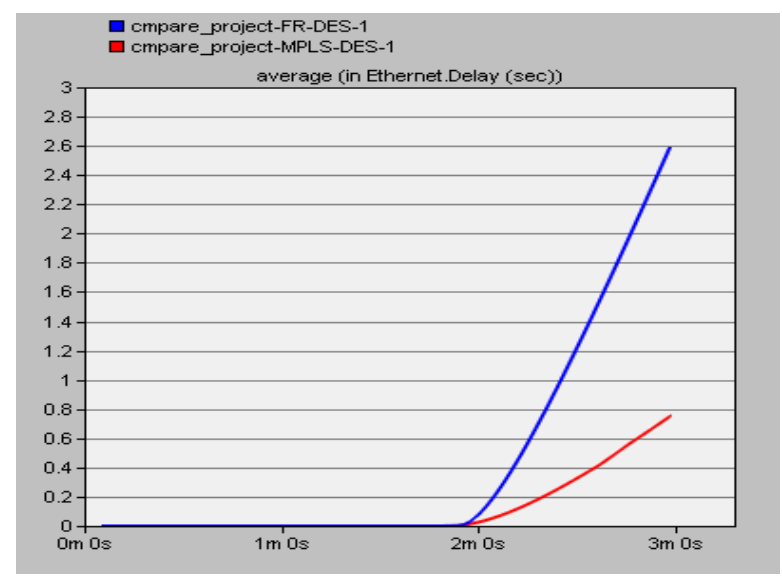

Fig.3: Ethernet Delay

For Ethernet delay we had the result that shown in figure 3. As y-axis (delay) the delay was decremented from 2.6 time unit in Frame-Relay to 0.8 time unit in MPLS which verified that MPLS is way more efficient than Frame-Relay in video conferences data transmission Because MPLS depends on label forwarding for packets rather than packet switching or other technologies that makes the network convergence and other specifications more useful to forward based labels rather than looking at the whole routing tables.

\subsection{End to End Delay}



Fig.4: End to End Delay

From network administrators point of view they seek to reduce latency in the whole network because latency makes delay in data movement at the whole path from the source to the distention so MPLS speed up the network six time better than Frame-Relay as the simulation result shown in figure 4.

\subsection{Traffic Received}

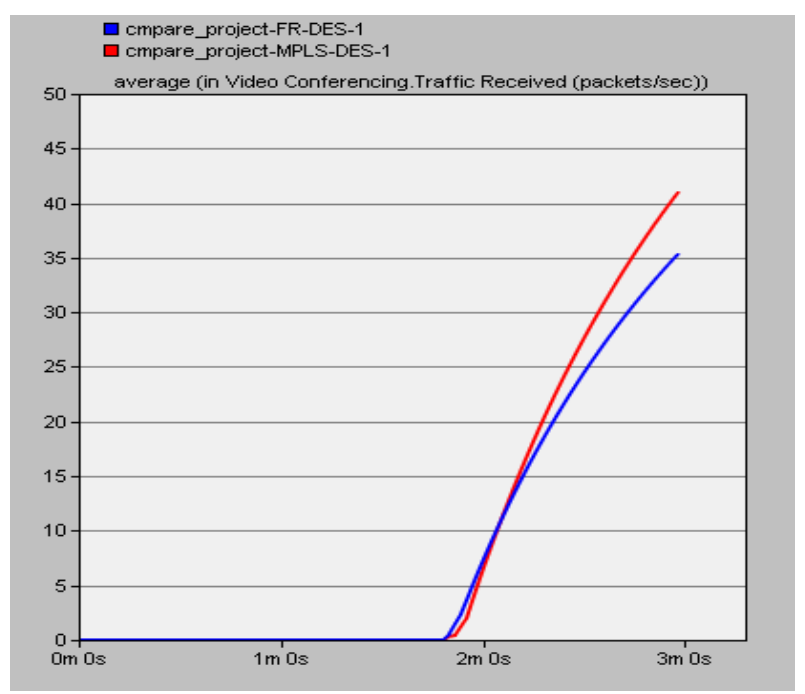

Fig.5: Traffic received

One of the parameters that can influence on overall performance of the Network is traffic received. Traffic received determines the amount of the data received at the destination. The traffic received or traffic dropped in applications such as video conferencing is often caused by the buffer overflow and the amount of data dropped can be determined from the amount of data transmitted and received. From the Figure 2 the traffic received for both MPLS and Frame-Relay increases linearly. There is an observable increase in the case of MPLS compared to Frame-Relay .For the results that we had we could notice the power of MPLS in packets received appeared when duration increased .These results and others led the mobile service provider move to the IP/MPLS networks for it's stability and high quality for Long Term Evolution (LTE) services. 


\section{CONCLUSION}

In the proposed system, the compared scenarios in OPNET Modeler 14.5 were evaluated. End-to-End delay (latency), Ethernet delay and traffic received were evaluated for the same topology using Frame-Relay and MPLS based on video conferencing as a load for our topology. This research had shown that enterprises and service providers can experience an improvement in the rate of achievement of business targets by implementing and maximizing the capabilities of MPLS in their networks. The service providers are suffering from the huge routing table; this can be solved by MPLS because Instead of forwarding packets based on IP address we will have router forwarding packets based on labels in these packets. By using MPLS based on labels instead of doing the layer 3 lookup for IP address we can get a lot more performance by using labels . Every single router will generate a local label for every network they have in IP routing table; these labels are existing in a table called Label Information Based (LIB). The cores of some mobile networks had been migrated to an MPLS infrastructure, providing a common platform for transport of both mobile voice and mobile data services, while Frame-Relay is limited to the standard of the congestion control process. MPLS use backhaul technology as a solution to the bottleneck in today's mobile network offers benefits and cost efficiencies in both legacy mobile backhaul and for future environments based on new technologies such as LTE. New services can be successfully rolled out, while mobile operators are able to leverage further cost benefits by using an MPLS-based in backhaul networks to deliver many non-backhaul services. The advantages of MPLS are many in addition to protocol neutrality, MPLS is highly scalable and can intelligently route time-sensitive voice and video packets through low-latency routes throughout the network. Tthese key features MPLS has been widely adopted by enterprises for their WANs, as the most recent data from Numerates Research indicates that around $84 \%$ of companies are now using MPLS for their WANs.

\section{REFERENCES}

[1] Daugherty B.; Metz C.," Multiprotocol label Switching and IP, Part1: MPLS VPNs over IP Tunnels". IEEE Internet Computing.

[2] Pultz, Richard., 2004., Analysis of MPLS-Based IP VPN Security: Comparison to Traditional L2VPNs such as ATM and Frame Relay, and Deployment Guidelines.

[3] Ghein, Luc De., 2007., MPLS Fundamentals.

[4] Rick Gallaher's MPLS Training Guide: Building Multi Protocol Label Switching Networks Paperback November 20, 2003, Syngress, Rick Gallaher

[5] "ISDN and Broadband ISDN with Frame Relay and ATM “ (4th Edition) ,October 25, 1998 , William Stallings

[6] Gurpreet Kaur \& Dinesh Kumar. “ MPLS Technology on IP Backbone Network "International Journal of Computer Applications, August 2010 .

[7] Rissal Efendi . "A Simulation Analysis of Latency and Packet Loss on Virtual Private Network through Multi Virtual Routing and Forwarding " International Journal of Computer Applications, December 2012

[8] Fang L.; Bita N.; Le R.; Miles J., "Interprovider IP- MPL Sservices: requirements, implementations, and Challenges". Communications Magazine, IEEE, vol.43, no.6, pp.119-128,June 2005 .

[9] Ramakrishnan, V.; Wargo, C.; John, S.,"GMPLS Network Security: Gap Analysis". ICNS Conference, 2008 IEEE Systems, pp. 1-7, 5-7 May 2008. 